TITLE:

\title{
Nonlinear rheological behavior associated with structural transitions in block copolymer solutions via nonequilibrium molecular dynamics
}

\author{
$\operatorname{AUTHOR}(\mathrm{S}):$ \\ Rychkov, I; Yoshikawa, K
}

\section{CITATION:}

Rychkov, I ... [et al]. Nonlinear rheological behavior associated with structural transitions in block copolymer solutions via nonequilibrium molecular dynamics. JOURNAL OF CHEMICAL PHYSICS 2004, 120(7): 3482-3488

\section{ISSUE DATE:}

2004-02-15

URL:

http://hdl.handle.net/2433/49854

\section{RIGHT:}

Copyright 2004 American Institute of Physics. This article may be downloaded for personal use only. Any other use requires prior permission of the author and the American Institute of Physics. 


\title{
Nonlinear rheological behavior associated with structural transitions in block copolymer solutions via nonequilibrium molecular dynamics
}

\author{
Igor Rychkov a) and Kenichi Yoshikawa ${ }^{\text {b) }}$ \\ Department of Physics, Graduate School of Science, Kyoto University, Kyoto 606-8502, Japan
}

(Received 9 June 2003; accepted 21 November 2003)

\begin{abstract}
The nonequilibrium molecular dynamics computer simulation method was used to study microsegregated block copolymer systems in a selective solvent under a shear flow field. Two polymer concentrations were considered, 0.3 and 0.4 , corresponding to the body centered cubic spherical and hexagonal cylindrical zero-shear phases, respectively. As the shear rate increased, both systems exhibited two-stage shear thinning, a peak in the scalar pressure, and normal stress differences. Microscopic connections were investigated by calculating the gyration and bond orientation tensors and the interaction energies per particle. At high shear rates, polymer chains elongate and orient along the direction of shear, and this is accompanied by the breaking-up of domains. The structure-rheology relation was discussed with regard to the morphological changes reported in our last study for the same systems. In particular, the structurally relevant critical values of the shear rate were found to delimit different behaviors of the shear rate-dependencies obtained in this work. (C) 2004 American Institute of Physics. [DOI: 10.1063/1.1642589]
\end{abstract}

\section{INTRODUCTION}

A liquid far out of equilibrium, e.g., subjected to shear flow, is commonly encountered and should be understood better. A suitable computer simulation technique is the nonequilibrium molecular dynamics (NEMD) method, which is an adaptation of the usual molecular dynamics method for sampling from nonequilibrium ensembles by applying external perturbation. ${ }^{1-3}$ Not only does this approach provide a route to transport coefficients in the limit of zero-applied field (so-called linear response), as an alternative to the Green-Kubo method used in conventional MD (with unfavorable signal to noise ratios), but it also makes it possible to investigate nonlinear phenomena through the use of larger perturbations.

Molecular systems under shear flow have been studied extensively by NEMD to find the corresponding transport coefficient, the shear viscosity. The nonlinear behavior of shear viscosity, such as shear thinning and shear thickening, is an important topic in materials science. Planar shear flow, or Couette flow, with kinetics of $\mathbf{u}=\mathbf{i}_{x} \gamma y$ can be generated in a NEMD simulation in several different ways. Computer simulations of small systems aimed at studying bulk properties, rather than surface effects, usually adopt periodic conditions on the boundaries of the simulation cell. Wall-driven flows, where the periodic boundary conditions (PBC) in one direction are replaced by hypothetical moving solid walls, do not allow for the study of bulk liquids free from the effects of the walls. A homogeneous procedure where an external space periodic perturbation field consistent with $\mathrm{PBC}$ acts on each particle makes it possible to compute a wave vectordependent viscosity from the resulting velocity profile. Such a field is synthetic in the sense that it does not exist in nature,

\footnotetext{
${ }^{\text {a)}}$ Electronic mail: rych@chem.scphys.kyoto-u.ac.jp

${ }^{b}$ URL: http://www.chem.scphys.kyoto-u.ac.jp
}

but rather is a mathematical device used to transform a difficult problem with boundary conditions into a much simpler mechanical problem. Taking this approach a step further, it is possible to show that the boundary conditions that correspond to planar shear flow can be incorporated homogeneously into the equations of motion. These equations are known as the SLLOD equations and must be coupled with the sliding-image Lees-Edwards PBC, so that a linear velocity profile is established throughout all of the image cells. The Lees-Edwards PBC alone can be used to set up and maintain a steady linear velocity profile, if the Reynolds number is sufficiently small so that microturbulence does not occur. However, this algorithm has several disadvantages, e.g., lack of contact with response theory and inability to study time-dependent flow.

The conventional MD method takes samples from the microcanonical ensemble. To model a system in the canonical ensemble, a procedure has to be found to keep the temperature constant. One approach is to modify the equations of motion so that the kinetic temperature is an integral of motion. To derive such equations, one can use Gauss' principle of least constraint. The resulting Gaussian thermostat rigidly builds a constant kinetic energy into equations of motion. As can be shown, ${ }^{4}$ the partition function of the Gaussian isokinetic ensemble is still separable and its configurational part is the same as that of the standard canonical ensemble. Thus, all position-dependent equilibrium properties of the two ensembles will be identical, whereas the dynamics is of course different from that in the Newtonian case.

When applied to the SLLOD equations, the Gaussian thermostat also serves to remove the heat produced by the shear field, without which it would be impossible to achieve a steady state. A side-effect of the Gaussian thermostat in this case is that it acts as a "profile-biased" thermostat because it interprets any deviations from laminar flow as thermal fluc- 
TABLE I. Structural behavior of diblock copolymers in selective solvents under shear flow depending on the shear rate (Ref. 17).

\begin{tabular}{|c|c|c|c|}
\hline Shear rate & 0 & 0.05 & 0.4 \\
\hline $\begin{array}{l}\text { bcc micelles } \\
\text { or } \\
\text { HEX cylinders }\end{array}$ & $\begin{array}{c}\text { Domain rearranging into } \\
\text { parallel layers }\end{array}$ & $\begin{array}{l}\text { Domain melting and } \\
\text { transition to } \\
\text { perpendicular lamellae }\end{array}$ & $\begin{array}{l}\text { Perfect perpendicular } \\
\text { lamellae; reduction in } \\
\text { lamellar spacing }\end{array}$ \\
\hline
\end{tabular}

tuations which should be suppressed. However, this effect becomes noticeable only in greatly nonlinear regimes, at extreme and experimentally unrealistic shear rates. Another issue arises when the Gaussian thermostat is applied to a molecular system atomistically, i.e., without acknowledging the saturating and unbreakable nature of bond interaction. The Gaussian atomic thermostat treats the shear-induced rotation of bonded monomeric units as deviations to be suppressed. However, this effect becomes significant only at higher shear rates $\left(\gamma \geqslant 2\right.$ in reduced units, see below) ${ }^{5,6}$

NEMD techniques have been successfully applied to both atomic and molecular systems. ${ }^{1,7}$ One of the most important discoveries was the observation of a decrease in the shear viscosity as the shear rate increases (a well-known phenomenon seen in complex fluids as shear thinning) for simple liquids such as argon, and even for hard-spheres, which indicates the universality of the phenomenon. The physically relevant variable is the product $\gamma \tau^{*}$ of the shear rate and the system-characteristic relaxation time; when its magnitude becomes on the order of unity, fluid undergoes structural changes accompanied by shear thinning, or otherwise has to respond as an elastic solid. In simple fluids, this corresponds to a shear rate which is several orders of magnitude larger than the values reasonably attainable in laboratory experiments, whereas for complex fluids, such as polymer solutions, with much longer relaxation times, shear thinning is observed experimentally. It has also been shown that at high shear rates both atomic and molecular fluids exhibit a volume dilatancy, which in a NEMD simulation of NVT ensemble manifests itself as an increase in the pressure. ${ }^{6,8}$ On the other hand, polymer systems have been found to show shear compression. ${ }^{9,10}$ At high shear rates, normal stress differences, which are responsible for the Weissenberg rod-climbing effect, ${ }^{11}$ have been reported for polymeric systems. ${ }^{9}$ The effect of shear flow on chain conformations has also been investigated using NEMD methods. ${ }^{9}, 12$ In a strong enough flow, chain molecules elongate and preferentially orient along the direction of shear.

We present here the results of a NEMD-SLLOD simulation of a more complex fluid, microphase-segregated block copolymers ${ }^{13-16}$ in a selective solvent. Block copolymers are taken at two different concentrations: one corresponding to a body centered cubic (bcc) lattice of spherical micelles (0.3) and the other to hexagonally (HEX) packed cylinders (0.4). As has been shown in Ref. 17, subjected to shear flow, such systems undergo structural changes. In weak flow, the domains are rearranged into new lattices, with the most densely packed crystallographic planes sliding in parallel to the shear plane (with the normal oriented along the shear gradient direction), whereas under stronger shear, significant domain breakup and remelting occur, leading to the formation of perpendicular lamellae, see Table I. In this article, we present the numerical results for the corresponding changes in rheological and microscopic properties.

\section{MODEL AND METHOD}

\section{A. Model potentials}

Using the bead-spring model, we studied systems of $n$ symmetrical diblock copolymer chains with $N=N_{A}+N_{B}$ beads per chain and a composition $f \equiv N_{A} / N=0.5$. The selectivity of the solvent is modeled using different parameters for the Lennard-Jones (LJ) potential. The bead pairs $A-A$ and $A-B$ interact via purely repulsive, truncated at the minimum and shifted LJ potential (Weeks-Chandler-Andersen potential),

$$
U_{\mathrm{LJ}}^{\mathrm{rep}}= \begin{cases}4 \epsilon\left[\left(\frac{\sigma}{r}\right)^{12}-\left(\frac{\sigma}{r}\right)^{6}+\frac{1}{4}\right], & r \leqslant 2^{1 / 6} \sigma \\ 0, & r \geqslant 2^{1 / 6} \sigma .\end{cases}
$$

Since $\epsilon$ can be used to set the energy scale and $\sigma$ can be used for the length scale, we will henceforth use so-called Lennard-Jones reduced units where $\epsilon=\sigma=1$ and also set the mass $m$ of the particles to unity, so that time is measured in units of $\left(\sigma^{2} m / \epsilon\right)^{1 / 2} \cdot B-B$ interaction includes an attractive part and is modeled by the following form of the LJ potential, modified so as to satisfy the continuity and smoothness requirements at the truncation point,

$$
U_{\mathrm{LJ}}=\left\{\begin{array}{ll}
\alpha \phi\left[\frac{1}{r^{12}}-\frac{1}{r^{6}}+\right. & \\
\left.\frac{r^{2}}{r_{c}^{2}}\left(\frac{6}{r_{c}^{12}}-\frac{3}{r_{c}^{6}}\right)-\frac{7}{r_{c}^{12}}+\frac{4}{r_{c}^{6}}\right], & r \leqslant r_{c} \\
0, & r \geqslant r_{c}
\end{array} .\right.
$$

The radius of interaction is chosen to be sufficiently small, $r_{c}=2$, to avoid possible "freezing" of multiparticle aggregates and to increase the speed of calculation. The potential has a minimum at $r \approx 2^{1 / 6} ; \alpha=4.913708224794938$ gives $\phi$ as the well depth. We take the temperature and well depth as $T=1$ and $\phi / T=1.2$, which implies that beads $A$ and $B$ are in favorable and weakly unfavorable solvent conditions, respectively.

For any two adjacent beads along the chain, in addition to the excluded volume LJ potential, Eq. (1), the attractive 


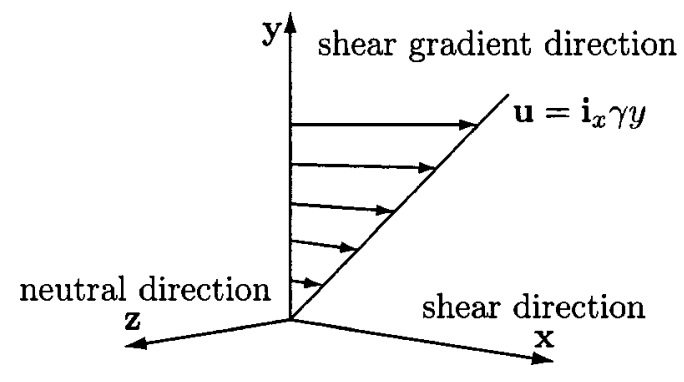

FIG. 1. Geometry and kinematics of the planar shear flow.

anharmonic finitely extensible nonlinear elastic (FENE) spring potential is also used with spring constant $k=30$ and maximum extension $R_{0}=1.5$,

$$
U_{\mathrm{FENE}}= \begin{cases}-\frac{1}{2} k R_{0}^{2} \ln \left[1-\left(\frac{r}{R_{0}}\right)^{2}\right], & r \leqslant R_{0} \\ \infty, & r \geqslant R_{0} .\end{cases}
$$

\section{B. Equations of motion}

We model the systems under conditions of steady planar shear flow (viscometric flow). ${ }^{11}$ The geometry and kinetics of the flow are illustrated in Fig. 1. The phase space is sampled by integrating the thermostated SLLOD equations of motion ${ }^{2}$ for each particle $i \in[1, n N]$,

$$
\begin{aligned}
& \dot{\mathbf{q}}_{i}=\mathbf{p}_{i} / m+\mathbf{i}_{x} \gamma q_{y i}, \\
& \dot{\mathbf{p}}_{i}=\mathbf{F}_{i}-\mathbf{i}_{x} \gamma p_{y i}-\lambda \mathbf{p}_{i}, \\
& \dot{d}_{x}(t)=\gamma,
\end{aligned}
$$

where the position of the particle $i$ is $\mathbf{q}_{i}\left(q_{x i}, q_{y i}, q_{z i}\right)^{T}$, the momentum is $\mathbf{p}_{i}\left(p_{x i}, p_{y i}, p_{z i}\right)^{T}, \mathbf{F}_{i}$ is the force acting on the $i$ th particle, and $d_{x}$ is the lattice strain associated with the Lees-Edwards periodic boundary conditions. The parameter $\lambda$ is the Gaussian thermostat multiplier,

$$
\lambda=\frac{1}{2 m K_{0}} \sum_{i=1}^{n N}\left[\mathbf{F}_{i} \cdot \mathbf{p}_{i}-\gamma p_{x i} p_{y i}\right] \text {. }
$$

The Gaussian thermostat fixes the instantaneous kinetic energy along a trajectory, $K(t)=1 / 2 m \sum_{i=1}^{n N} \mathbf{p}_{i} \cdot \mathbf{p}_{i}=K(0) \equiv K_{0}$. If the popular Gear predictor-corrector method ${ }^{18,19}$ is used to numerically integrate such equations of motion, the kinetic energy tends to drift away from its initial value. Therefore, a newer symplecticlike integrator obtained in Ref. 20 using an operator-splitting technique is employed, with the integration time step as large as 0.01 , which is one order of magnitude greater than in the Gear method.

\section{Technical details}

Simulations were performed in a cubic cell of size $L$ $=40$ with periodic boundary conditions for systems of symmetrical diblock copolymers with a fixed chain length of $N$ $=10+10$. Systems with different concentrations in the range $\rho=0.2-0.8$ were simulated to obtain a zero-shear phase diagram. The effect of shear flow on systems with two concentrations was considered: $\rho \equiv n N / L^{3}=0.3$ with the number of chains $n=960$, and $\rho=0.4$ with $n=1280$. Without flow, the first block copolymer system corresponds to a bcc lattice of spherical micelles $(S)$, and the second to hexagonally packed cylinders $(C) .{ }^{17}$ For each shear rate, simulations were carried out from a random configuration until the time dependencies of energy and other variables of interest appeared to be constant. The typical times $t_{0}$ required to reach a steady state varied from as long as $t_{0}=6 \times 10^{4}$ at low shear rates to $t_{0}$ $=4 \times 10^{3}$ at the highest shear rate $\gamma=2$.

Solvent molecules surrounding block copolymers are not simulated explicitly in our model. Instead, the main phenomenological effects of the medium such as shear impulse transfer and thermostating are included in the equations of motion, and selectivity is ensured by the effective potentials. Such a mean-field approach to the medium corresponds to the situation when the time and space scales of processes in the solvent are much smaller than those of the solute. Thus, the values calculated represent the corresponding polymer contributions.

\section{Pressure tensor}

The key quantity to be calculated in theoretical studies of the rheology of shear flow is the pressure tensor. ${ }^{21} \mathrm{We}$ calculated the pressure tensor in the atomic representation, which is given by a sum of site-site contributions,

$$
\mathbf{P}=\frac{1}{V}\left(\sum_{i}^{n N} \frac{\mathbf{p}_{i} \mathbf{p}_{i}}{m_{i}}+\sum_{j>i}^{n N} \mathbf{r}_{i j} \mathbf{F}_{i j}\right),
$$

where $\mathbf{F}_{i j}$ is the force exerted on bead $i$ by bead $j, \mathbf{r}_{i j} \equiv \mathbf{r}_{i}$ $-\mathbf{r}_{j}$ is the distance between the particles, and $m_{i}, \mathbf{r}_{i}, \mathbf{p}_{i}$ are the mass, position, and impulse of particle $i$; the vector pairs are dyadic products. Group-based, ${ }^{22}$ e.g., "molecular," expressions for the pressure tensor are also possible, leading to the same average values but to hopefully smaller fluctuations, which gives better statistics. However, in the systems studied, the block copolymer chains also formed dynamically open aggregates, effectively creating new temporal bonds. This makes the grouping for a group-based pressure tensor, where fast fluctuations are filtered out, a nontrivial task. In test runs, the molecular pressure, for example, still had significant high-frequency fluctuations.

In steady planar shear flows, $\mathbf{u}=\mathbf{i}_{x} \gamma y$, the only nonvanishing components of the pressure tensor are the diagonal $P_{i i}$ and $P_{x y}=P_{y x}$. Therefore, all of the information is included in the isotropic scalar pressure $p$ and three viscometric functions,

$$
\begin{aligned}
& \eta=-\frac{P_{x y}}{\gamma}, \\
& N_{1}=P_{y y}-P_{x x}, \quad N_{2}=P_{z z}-P_{y y}, \\
& p=\frac{1}{3} \operatorname{Tr} \mathbf{P},
\end{aligned}
$$

where $\eta$ is the non-Newtonian shear viscosity, and $N_{1}, N_{2}$ are the first and second normal stress differences. 


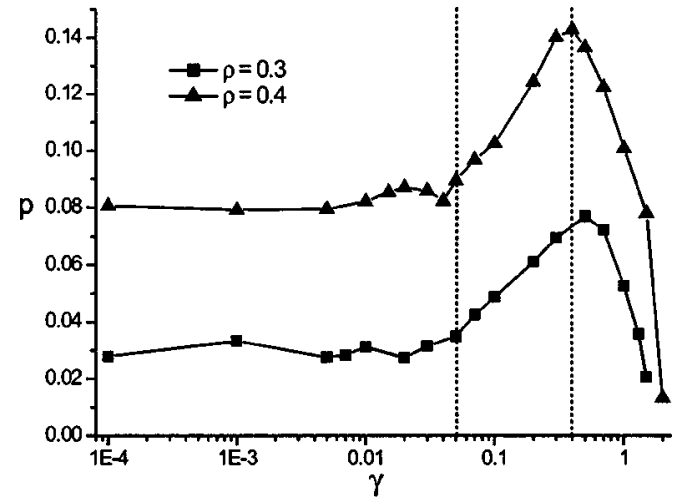

FIG. 2. Isotropic pressure vs shear rate for the two systems.

\section{RESULTS}

In the last article ${ }^{17}$ we showed that at shear rates larger than $\gamma^{*} \approx 0.05$, both systems undergo structural changes from parallel sliding layers to perpendicular lamellae. Perfect perpendicular lamellae are formed at $\gamma^{* *} \approx 0.4$; at $\gamma>\gamma^{* *}$, further changes include only reduction in lamellar spacing through the splitting of lamellae. In this article, we describe the dependence of rheological and microscopic properties on the shear rate for the systems studied in Ref. 17. It is natural to anticipate that the critical values $\gamma^{*}$ and $\gamma^{* *}$ should mark different behaviors in the corresponding figures presented below. All of the figures give the shear rate on a logarithmic scale along the $x$-axis, and, if not specified otherwise, the squares correspond to $\rho=0.3$ and the filled triangles to $\rho=0.4$; vertical dotted lines correspond to $\gamma=\gamma^{*}$ and $\gamma^{* *}$.

\section{A. Pressure}

The dependence of the isotropic scalar pressure $p$, Eq. (11), on the shear rate $\gamma$ is shown in Fig. 2. For both systems, the pressure increases in the interval $\gamma^{*}<\gamma<\gamma^{* *}$, reaches a maximum value at $\gamma^{* *}$, and then falls. To analyze this behavior, we can deduce from the formula for the pressure tensor, Eq. (8), that at a given temperature an increase in pressure means that repulsive forces become stronger, or the system is less "associative." For a decrease in pressure, under the usual experimental conditions with a constant ambient pressure, this would correspond to an increase in density, or compression. Conversely, structural changes that make the system more compressible should lead to a decrease in pressure in the canonical ensemble. Returning to our systems, as shown in Ref. 17, increasing the shear rate above $\gamma^{*}$ initiates the break-up of domains and their rearrangement into perpendicular lamellae, which are perfectly formed by $\gamma^{* *}$. Thus, within this transition region, the system becomes less associative. However, at $\gamma>\gamma^{* *}$, the chains elongate and are significantly oriented about the shear direction, thereby allowing finer packing, i.e., the system becomes more compressible.

\section{B. Viscosity}

The systems studied exhibit shear thinning, as can be seen from the dependence of the shear viscosity on the shear rate, Eq. (9), as shown in Fig. 3. The observed thinning pro-

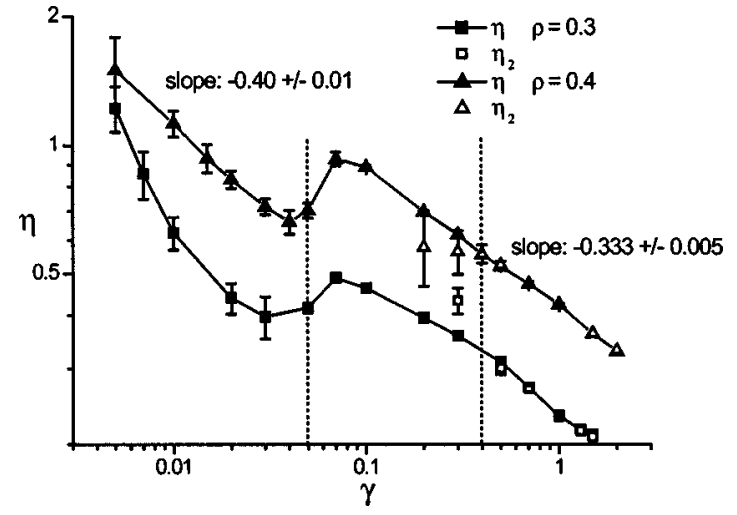

FIG. 3. Log-log plot of shear viscosity vs shear rate for the two systems. The viscosity obtained from the heat production-removal equality, Eq. (12), is shown with open symbols.

ceeds in two stages: initially there is a steep slope, and then, after some insignificant shear thickening, there is a second shear-thinning curve, somewhat less steep. These two regions correspond to the two different flow regimens: sliding parallel layers and the flow of perpendicular lamellae. Unfortunately, the methodology adopted does not easily allow to find the linear, Newtonian, regime at the lowest shear rates. This is because the atomistic pressure tensor component $P_{x y}$ has, due to bond oscillations, ${ }^{6}$ large standard deviations and, in the Newtonian regime, a near-zero mean value, $\mathrm{O}(\gamma)$, leading to large statistical uncertainties in $\eta$. The same rapidly fluctuating bond contributions to the atomic pressure tensor cause oscillatory behavior in the stress-stress autocorrelation function, which hampers attempts at obtaining zeroshear viscosity using the Green-Kubo relation. Another value that suffers from large statistical errors at small shear rates is the shear viscosity $\eta_{2}$ obtained from the heat production-removal equality, ${ }^{2}$

$$
\eta_{2}=\frac{3 T \rho \lambda}{\gamma^{2}}
$$

This is, in principle, the same physical quantity as that in Eq. (9), however its reliable estimates are attained only at rather high shear rates, see Fig. 3.

\section{Normal stress differences}

Polymeric liquids under shear flow have normal stress differences, which are responsible for several visually striking effects, such as rod-climbing: the polymer will climb up an inserted rotating rod. ${ }^{21}$ The first normal stress difference, Eq. (10), is practically always positive and numerically much larger than the usually negative second normal stress difference, which leads to a strengthening of the inequality $P_{y y}$ $>P_{z z} \gg P_{x x}$. The same conclusions can be drawn for the block copolymer systems studied, as seen from Fig. 4 . The figures also show that the increase in $N_{1}$ with increasing $\gamma$ begins at $\gamma \approx \gamma^{*}$ and becomes greater at $\gamma>\gamma^{* *}$. Also, since this is a polymeric effect, the normal stress differences are naturally more pronounced for systems with a greater polymer concentration, which is indeed the case for our systems. A simple structural explanation of the existence of the first 


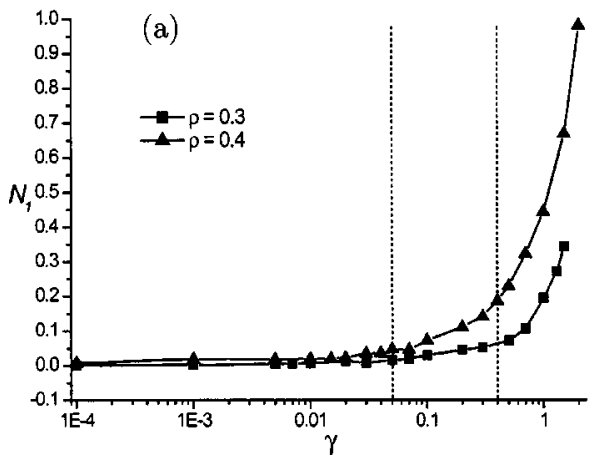

normal stress difference is that extra tension (a negative contribution to pressure) arises along the streamlines which the chain molecules stretch and align along: thermal motion makes the chain want to snap back. This explanation is supported by the results presented below, see Fig. 6(a) below, which show the corresponding changes in chain conformations.

\section{Interaction energy}

Figure 5(a) shows the volume (Lennard-Jones) interaction energy per particle. For the systems studied here, this value reflects the degree of system association, i.e., it is a measure of the participation of a particle in an aggregate. With this in mind, Fig. 5(a) suggests that at $\gamma=\gamma^{*}$, the domains begin to disintegrate, and this process is further enhanced at $\gamma>\gamma^{* *}$, although there may be an asymptotic plateau at $\gamma \geqslant 2$. The less intuitively clear dependence is that of the bond oscillation energy per monomer, presented in Fig. 5(b). Again, changes begin when the shear rate exceeds $\gamma^{*}$. The bond oscillation energy decreases, with an inflection point at approximately $\gamma^{* *}$, and reaches a plateau by $\gamma \approx 2$. This means that the formation of perpendicular lamellae leads to the chain bonds being less stretched. Such chain "relaxation" can be caused by the breaking-up of the domains and a decrease in domain curvature.

\section{E. Chain conformations}

Although chain bonds relax under shear flow, chains as a whole elongate and also orient along the shear direction. To study the changes in chain conformations, two tensor quantities $^{12}$ were calculated for beads of both types for the system 0.3 . One is the gyration tensor, defined as follows:

$$
\mathbf{G}=\frac{1}{n} \sum_{a=1}^{n} \frac{1}{N} \sum_{i=1}^{N}\left(\mathbf{r}_{a i}-\mathbf{r}_{a}\right)\left(\mathbf{r}_{a i}-\mathbf{r}_{a}\right)
$$

where $\mathbf{r}_{a} \equiv 1 / N \sum \mathbf{r}_{a i}$ is the center of mass of a polymer. The other is the bond orientation tensor,

$$
\mathbf{O}=\frac{3}{2 n} \sum_{a=1}^{n} \frac{1}{N-1} \sum_{i=1}^{N-1} \frac{\left(\mathbf{r}_{a i+1}-\mathbf{r}_{a i}\right)\left(\mathbf{r}_{a i+1}-\mathbf{r}_{a i}\right)}{b^{2}}-\frac{1}{2} \mathbf{I},
$$

where $\mathbf{I}$ is the unit tensor and $b$ is the mean bond length, which for the potentials used is $\approx 1$. In accordance with the geometry of shear flow, a symmetry consideration requires that for the elements of both tensors $T_{x z}=T_{z x}=T_{y z}=T_{z y}$ $=0$, which was confirmed in our simulations, and therefore just three diagonal elements, $T_{x x}, T_{y y}$, and $T_{z z}$, provide enough information. Figure 6(a) shows the dependence of the diagonal elements of the gyration tensor on the shear rate for beads of type $A$ and $B$. Increasing the shear rate much above $\gamma^{*}$ leads to the following inequalities: $G_{x x} \gg G_{z z}$ $>G_{y y}$, which means that the blocks elongate and orient along the shear direction. Naturally, $B$-blocks elongate less because these blocks are still in the condition of a poor solvent. Furthermore, $\gamma=\gamma^{* *}$ is roughly the inflection point for all of the curves in Fig. 6(a), which suggests the existence of a plateau at some higher shear rates. It is worth noting that not only do changes occur in the $(x y)$ plane but that also $G_{z z}$ decreases at higher shear rates, which provides a microscopic explanation for the shear-induced decrease in the spacing of perpendicular lamellae observed in Ref. 17. The diagonal elements of the bond orientation tensor are shown in Fig. 6(b). Again, starting from $\gamma^{*}$, there is ever-increasing asymmetry, which for this tensor means that bonds are significantly oriented along the shear direction, and this effect is
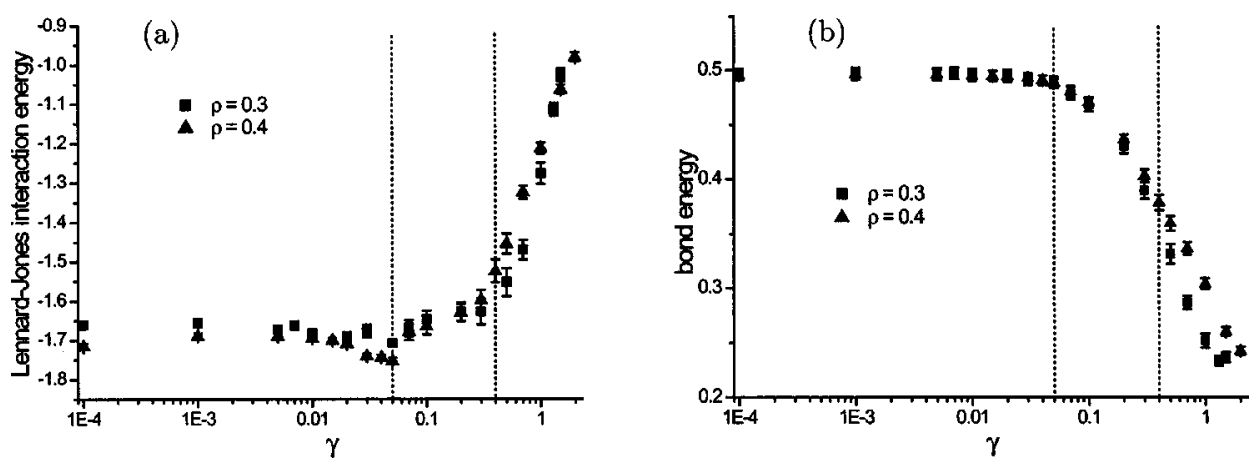

FIG. 5. Lennard-Jones (a) and bond oscillation energy (b) per particle. 

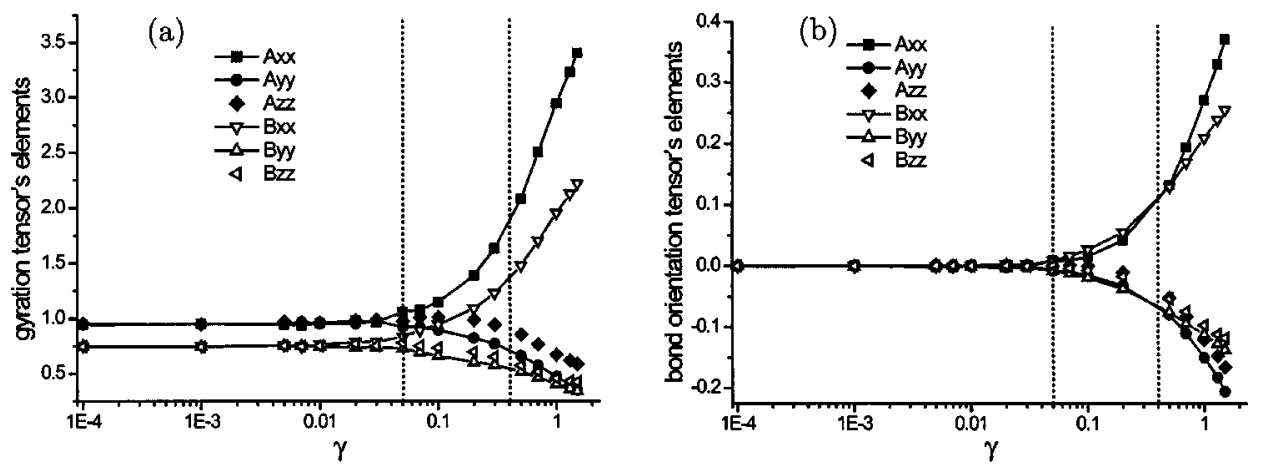

FIG. 6. Components of gyration (a) and bond orientation (b) tensors for $A$-beads and $B$-beads (triangles) for the system 0.3 .

also somewhat more pronounced for $A$-bonds. Similar graphs were obtained for both tensors for the system 0.4 .

\section{CONCLUSIONS}

This work follows a previous one ${ }^{17}$ by the authors and describes the rheological and microscopic aspects of the same systems. In the last work, the structural changes that the systems undergo with an increase in the shear rate were illustrated, and the existence of two critical values of the shear rate were identified. The first value marks the end of the flow regime as the sliding of parallel micellar layers and the onset of the formation of perpendicular lamellae. The second value, which is one order of magnitude larger than the first value, marks the end of this phase transition. The second critical shear rate corresponds to the appearance of perfectly formed perpendicular lamellae and with a further increase in the shear rate the only structural changes include a shear-induced reduction in lamellae spacing, with no further phase transitions in the shear rate interval considered. ${ }^{17}$ Not surprisingly, we were very interested in investigating whether these values have any special meaning with regard to rheological and microscopic properties. Indeed, these values appear to delimit different behaviors in the corresponding graphs. Except for the shear viscosity, all of the quantities studied show little or no dependence on shear rates less than the first critical value. Greater shear rates lead to such non-Newtonian phenomena as pressure dilatancy and normal stress differences; the microscopic properties show evidence of domain disintegration, and chain elongation and orientation along the shear direction. The second critical value of the shear rate is the maximum point of isotropic pressuredependence that may be a turning-point in the competition between shear-induced domain dissociation, which increases the pressure, and chain packing, which decreases it. The second critical value of the shear rate is the inflection point in graphs of interaction energies and the diagonal elements of the gyration tensor, which implies the existence of a plateau in these graphs at greater shear rates and a limit in the shearinduced changes in these values.

Perhaps the most important rheological property, shear viscosity, does not remain constant (in fact the Newtonian regime, which is inherently small for polymers, was not even found in this work because of the large statistical uncertainties at low shear rates), but rather decreases even before the first critical shear rate. However, increasing the shear rate above this value breaks the monotonic character of shear thinning: the shear viscosity rises and then the second "stage" of shear thinning begins. The crossover delimits two different structures and therefore two different patterns for releasing the stress in the system: the sliding of parallel close-packed layers and the formation of perpendicular lamellae.

Interestingly, similar dependencies are seen for the systems of $\rho=0.3$ and $\rho=0.4$, even though the two systems have very different morphologies in the absence of shear flow, i.e., bcc micelles and HEX cylinders. This suggests the universality of the parallel-perpendicular phase transition and the corresponding rheological behavior in block copolymer systems in selective solvent under shear flow, regardless of the concentration.

Although the method used here allowed us to draw some physically interesting conclusions consistent with previous reports, there is of course room for further developments. The problem of low statistical certainty in determining the Newtonian shear viscosity can probably be solved by some preaveraging of the fast oscillations in the pressure tensor. The issue of the applicability of the atomistic thermostat can be avoided by incorporating a configurational temperature thermostat, ${ }^{5}$ which would also allow deviations from a fixed linear velocity profile, i.e., richer hydrodynamics. The possible effects of periodic boundary conditions on the structures formed should also be investigated. These points are currently being studied by the authors. Many of the physical conclusions and explanations in this paper are somewhat speculative. A more theoretically sound treatment is also underway.

${ }^{1}$ D. M. Heyes, The Liquid State: Applications of Molecullar Simulations (Wiley, Great Britain, 1998).

${ }^{2}$ D. J. Evans and G. P. Morriss, Statistical Mechanics of Nonequilibrium Liquids (Academic, London, 1990).

${ }^{3}$ M. P. Allen and D. J. Tildesley, Computer Simulation of Liquids (Oxford University Press, London, 1989).

${ }^{4}$ P. Minary, G. J. Martyna, and M. E. Tuckerman, J. Chem. Phys. 118, 2510 (2003).

${ }^{5}$ J. Delhommelle and D. J. Evans, J. Chem. Phys. 115, 43 (2001).

${ }^{6}$ S. T. Cui, P. T. Cummings, and H. D. Cochran, J. Chem. Phys. 104, 255 (1996).

${ }^{7}$ S. Hess, C. Aust, L. Bennett, M. Kröger, C. P. Borgmeyer, and T. Weider, Physica A 240, 126 (1997).

${ }^{8}$ D. J. Evans, Physica A 118, 51 (1983).

${ }^{9}$ Z. Xu, J. J. de Pablo, and S. Kim, J. Chem. Phys. 102, 5836 (1995).

${ }^{10}$ M. Kröger, W. Loose, and S. Hess, J. Rheol. 37, 1057 (1993).

${ }^{11}$ N. Phan-Thien, Understanding Viscoelasticity (Springer-Verlag, Berlin, 2002). 
${ }^{12}$ Flexible Polymer Chain Dynamics in Elongational Flow, edited by T. Q. Nguyen and H.-H. Kausch (Springer-Verlag, Berlin, 1999).

${ }^{13}$ F. S. Bates and G. H. Fredrickson, Phys. Today 52, 32 (1999).

${ }^{14}$ I. W. Hamley, The Physics of Block Copolymers (Oxford University Press, London, 1999).

${ }^{15}$ T. P. Lodge, B. Pudil, and K. J. Hanley, Macromolecules 35, 4707 (2002).

${ }^{16}$ G. H. Fredrickson, Annu. Rev. Mater. Sci. 26, 501 (1996).

${ }^{17}$ I. Rychkov and K. Yoshikawa (2003), Macromolecular Theory \& Simulations (to be published).
${ }^{18}$ W. Gear, Numerical Initial Value Problems in Ordinary Differential Equations (Prentice-Hall, London, 1971).

${ }^{19}$ D. J. Evans and G. P. Morriss, Comput. Phys. Rep. 1, 297 (1984).

${ }^{20}$ F. Zhang, D. J. Searles, D. J. Evans, J. S. den Toom Hansen, and D. J. Isbister, J. Chem. Phys. 111, 18 (1999).

${ }^{21}$ R. B. Bird, R. C. Armstrong, and O. Hassager, Dynamics of Polymeric Liquids, 2nd ed. (Wiley, New York, 1987).

${ }^{22}$ P. H. Hünenberger, J. Chem. Phys. 116, 6880 (2002). 